\title{
Confinement of an electron in a non-homogeneous magnetic field: Integrable vs superintegrable quantum systems
}

\author{
A. Contreras-Astorga ${ }^{\mathrm{a}, \mathrm{b}}, \mathrm{J}^{\mathrm{N}}$ Negro $^{\mathrm{c}, *}, \mathrm{~S}$. Tristao ${ }^{\mathrm{c}}$ \\ a Department of Mathematics and Actuarial Science, Indiana University Northwest, 3400 Broadway, Gary, IN 46408, USA \\ b Departamento de Física, Cinvestav, A.P. 14-740, 07000 México D.F., Mexico \\ c Departamento de Física Teórica, Atómica y Óptica and IMUVA, Universidad de Valladolid, E-47011 Valladolid, Spain
}

\section{A R T I C L E I N F O}

\section{Article history:}

Received 16 June 2015

Received in revised form 30 August 2015

Accepted 1 September 2015

Available online 7 September 2015

Communicated by P.R. Holland

\section{Keywords:}

Dirac equation

Shape-invariance

Factorization method

Integrable systems

\begin{abstract}
A B S T R A C T
This paper deals with the problem of an electron in a non-homogeneous magnetic field perpendicular to a plane. From the classical point of view this is an integrable, but not superintegrable, solvable system. In the quantum framework of the Dirac equation this integrable system is solvable too; the energy levels and wavefunctions of bound states, for its reduction to the plane, are computed. The effective onedimensional matrix Hamiltonian is shown to belong to a shape-invariant hierarchy. Through this example we will shed some light on the specific properties of a quantum integrable system with respect to those characteristic of superintegrable systems.
\end{abstract}

(c) 2015 Elsevier B.V. All rights reserved.

\section{Introduction}

This work is devoted to a system that consists of an electron under an external magnetic field perpendicular to the $x-y$ plane. The magnetic field is non-uniform, its intensity behaving as the inverse of the distance to the $z$-axis. In these conditions the classical system is integrable, but not superintegrable. This system can be restricted to the plane $x-y$, and in this sense it is quite interesting to find its properties in the light of other well-known superintegrable systems, such as the Landau system of a constant perpendicular magnetic field or the planar Coulomb system. For instance, in our present situation there can exist bounded, although non-periodic, motions or exclusively unbounded motions depending on the sign of the angular momentum. As the trajectories and motion can be obtained in closed implicit form, we can say that the system is solvable.

In the quantum framework of the Dirac equation the system is solvable too, and for such above mentioned sign, the solutions to the eigenvalue problem will be obtained. As the system is solvable, it is investigated whether the reduced radial matrix Hamiltonian belongs to a shape-invariant Hamiltonian hierarchy. In this context, the matrix intertwining operators will be characterized as well as the symmetries of the hierarchy. This implies that the solutions

\footnotetext{
* Corresponding author.

E-mail addresses: alonso.contreras.astorga@gmail.com (A. Contreras-Astorga), jnegro@fta.uva.es (J. Negro), hetsudoyaguiu@gmail.com (S. Tristao).
}

can be obtained algebraically, by means of raising operators. We want to remark that we have found some new properties related to the matrix shape-invariance: (i) It can be realized by means of anti-intertwining operators, due to the fact that the Dirac equation has positive and negative eigenvalues; (ii) There is a wide freedom in the intertwining operators of the shape-invariance in the same hierarchy, in particular we have characterized a four parameter set of such operators; (iii) The symmetries of the shape-invariant hierarchy of matrix Hamiltonians are shown to play an important role.

We will remark the most important properties of the spectrum of bound states of this integrable system with respect to those of superintegrable quantum systems (see for instance the review of Ref. [1] on superintegrability). The most striking difference is that it consists of a dense set of non-isolated points while the known superintegrable systems have a set of isolated points as the discrete spectrum. Contrary to some general belief, the spectrum is highly degenerated, although the system is not superintegrable [1-3]. We will also show how this system is algebraically solvable, but the involved operators have some important differences to those corresponding to superintegrable systems.

This work will develop and extend some methods introduced in a previous paper for a different problem [4]. The present paper is organized as follows. The system is introduced in its classical version along Section 2 showing the features of the classical trajectories. The relativistic quantum system, in the frame of the Dirac equation, is analyzed in Section 3, where the discrete spectrum and eigenfunctions are obtained. In Section 4 the shape-invariant properties of the reduced radial matrix Hamiltonian are investi- 


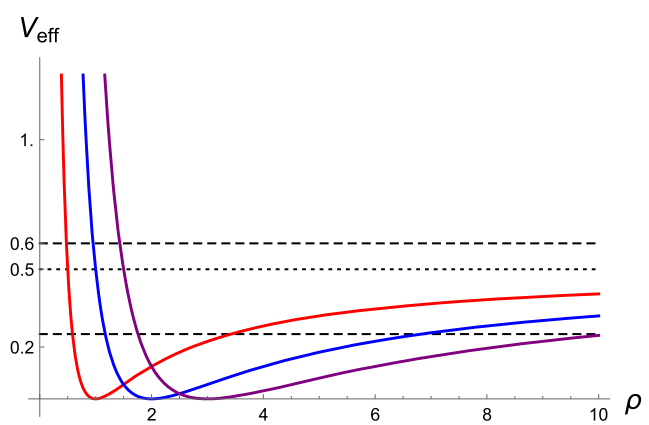

Fig. 1. Effective potential for $\ell=1,2,3$ and $m_{0}=c=k=e=1, \mu=0$. The dashing lines correspond to the energies $E=0.25$ (bound trajectory) and $E=0.6$ (unbounded trajectory). The dotted line separates the energies of bound and unbounded motions.

gated. Finally, Section 5 will be devoted to some remarks and conclusions.

\section{Classical motion}

We will consider an electron under the influence of a magnetic field with a rotational symmetry around the $z$-axis given by

$\mathbf{B}=\left(0,0, \frac{k}{\rho}\right)$

where $\rho=\sqrt{x^{2}+y^{2}}$ and $k$ is a non-vanishing constant. Its vector potential takes the expression

$\mathbf{A}=\frac{k}{\rho}(-y, x, 0)$

or $\mathbf{A}=k(-\sin \theta, \cos \theta, 0)$, in terms of the cylindrical coordinates $(\rho, \theta, z)$. Now, we want to describe the non-relativistic motion of an electron of mass $m_{0}$, and charge $e$ subject to this magnetic potential. The corresponding Hamiltonian using cylindrical coordinates has the following form

$H=\frac{P_{\rho}^{2}}{2 m_{0}}+\frac{\left(P_{\theta}-\frac{e k}{c} \rho\right)^{2}}{2 m_{0} \rho^{2}}+\frac{P_{z}^{2}}{2 m_{0}}$

where we recall the expressions of the canonical momenta

$\dot{\rho}=\frac{P_{\rho}}{m_{0}}, \quad \dot{\theta}=\frac{P_{\theta}}{m_{0} \rho^{2}}-\frac{e k}{m_{0} c \rho}, \quad \dot{z}=\frac{P_{z}}{m_{0}}$.

Since the coordinates $\theta$ and $z$ are cyclic the corresponding momenta will be constants of motion: $P_{\theta}=\ell$ (the angular momentum around $z$ ) and $P_{z}=\mu$ (the linear momentum along $z$ ). According to (4), this means that the velocity $\dot{z}$ will be constant but $\dot{\theta}$ will depend on the motion of $\rho$. After replacing these constants we are left with an effective Hamiltonian for the remaining variable $\rho$,

$$
\begin{aligned}
H_{\text {eff }}(\rho) & =\frac{P_{\rho}^{2}}{2 m_{0}}+\frac{\ell^{2}}{2 m_{0} \rho^{2}}-\frac{e k \ell}{m_{0} c \rho}+\frac{e^{2} k^{2}}{2 m_{0} c^{2}}+\frac{\mu^{2}}{2 m_{0}} \\
& \equiv \frac{P_{\rho}^{2}}{2 m_{0}}+V_{\text {eff }}(\rho)
\end{aligned}
$$

where the product $e k \ell$ must be positive if we want the effective potential $V_{\text {eff }}(\rho)$ to have a minimum and allow for bounded motions. A schematic plot of this potential for such a case can be seen in Fig. 1. A situation where $e k \ell<0$ is represented in Fig. 2, where $e=k=1$ and $\ell=-1,-2,-3$.

Thus, we have a classical system in a three dimensional space with three (independent) constants of motion: $H, P_{\theta}$ and $P_{z}$. This means that our system is integrable, but not superintegrable. The

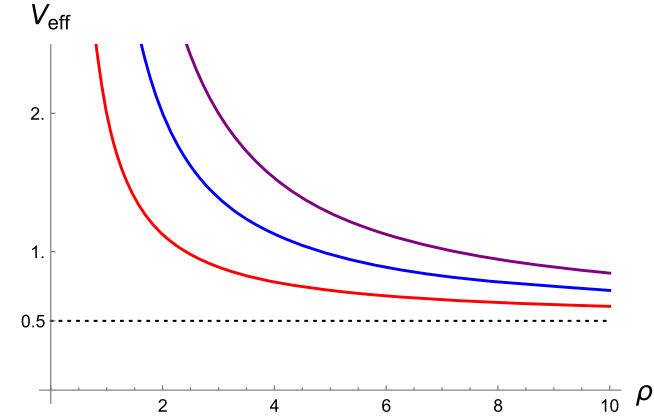

Fig. 2. Effective potential for $\ell=-1,-2,-3$ with the same values of the other parameters as in Fig. 1.

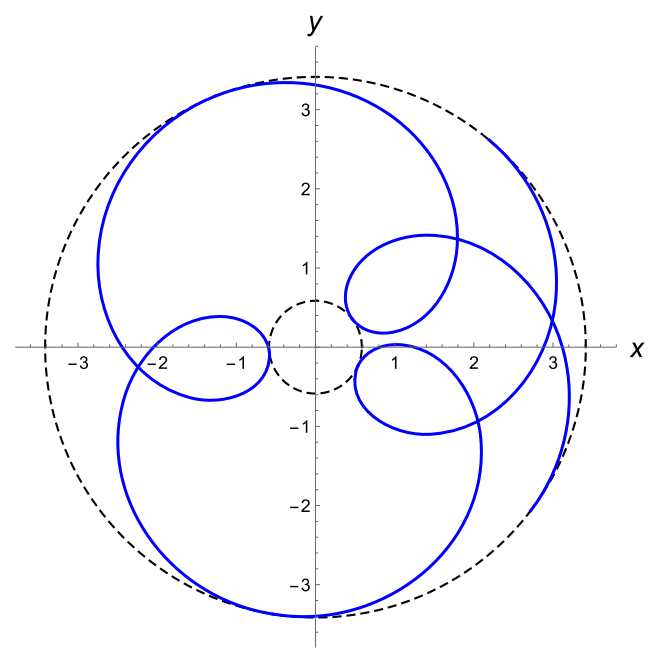

Fig. 3. Trajectory for the electron for $E=0.25$ in continuous line, bounded by the inner and outer circles in dashing lines. The values of the parameters are $\ell=1$, $\mu=0, m_{0}=1, c=1, k=1, e=1$.

equation of the projection of trajectory on the $x-y$ plane for an energy $E$ can readily be obtained from (4) and (5). If $\frac{\mu^{2}}{2 m_{0}} \leq E<$ $\frac{e^{2} k^{2}}{2 m_{0} c^{2}}+\frac{\mu^{2}}{2 m_{0}}$ this trajectory is bounded and the equation for such orbits is

$$
\begin{aligned}
\theta(\rho)= & -\arcsin \left(\frac{\ell / \rho-e k / c}{\sqrt{2 m_{0} E-\mu^{2}}}\right) \\
& -\frac{e k / c}{\sqrt{e^{2} k^{2} / c^{2}+\mu^{2}-2 m_{0} E}} \times \\
& \arcsin \frac{\left(e^{2} k^{2} / c^{2}+\mu^{2}-2 m_{0} E\right) \rho-\ell e k / c}{\ell \sqrt{2 m_{0} E-\mu^{2}}} .
\end{aligned}
$$

A graphic of this type of bounded trajectories on the $x-y$ plane is shown in Fig. 3. When $E>\frac{e^{2} k^{2}}{2 m_{0} c^{2}}+\frac{\mu^{2}}{2 m_{0}}$, the motion is unbounded and it is given by

$$
\begin{aligned}
\theta(\rho)= & -\arcsin \left(\frac{\ell / \rho-e k / c}{\sqrt{2 m_{0} E-\mu^{2}}}\right) \\
& -\frac{e k / c}{\sqrt{2 m_{0} E-e^{2} k^{2} / c^{2}-\mu^{2}}} \times \\
& \operatorname{arccosh} \frac{\left(2 m_{0} E-e^{2} k^{2} / c^{2}-\mu^{2}\right) \rho+\ell e k / c}{\ell \sqrt{2 m_{0} E-\mu^{2}}} .
\end{aligned}
$$

In Fig. 4 it is given the aspect of an unbounded trajectory. For both cases, the motion in the $z$-direction is uniform. The implicit time 


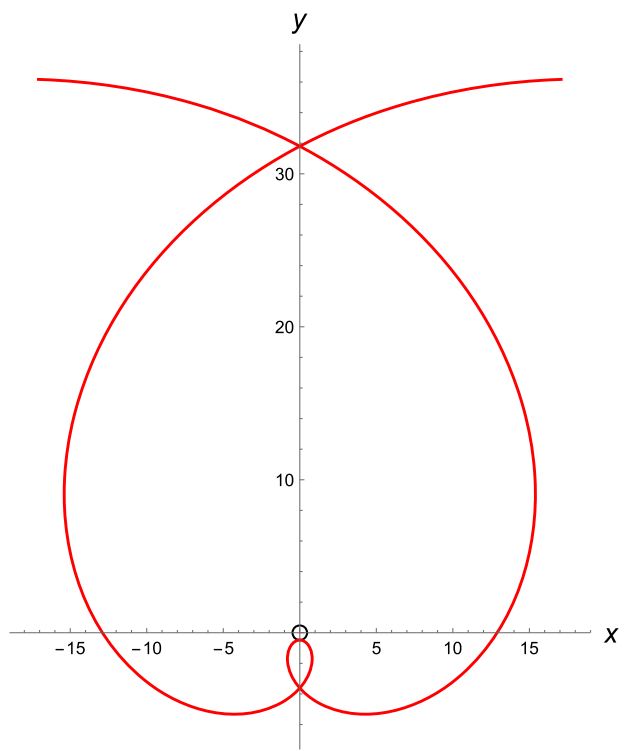

Fig. 4. Unbounded trajectory for $E=0.6$. The values of the other parameters are the same as in Fig. 3.

evolution $\rho(t)$ can also be obtained and has a similar expression. Thus, we could say that this is a solvable classical system.

\section{Quantum system: Dirac Hamiltonian}

We will consider this system in the context of quantum mechanics through the Dirac equation. We will show that in this framework the system is also solvable. However, the solutions have some striking properties worth to analyse.

\subsection{Separation of variables}

The Dirac Hamiltonian corresponding to the interaction of an electron with the above magnetic field is

$\mathbf{H}=c\left(\mathbf{P}-\frac{e}{c} \mathbf{A}\right) \cdot \boldsymbol{\alpha}+m_{0} c^{2} \boldsymbol{\beta}$

where $e$ is the electron charge, $m_{0}$ its rest mass, $\mathbf{P}=-i \hbar \nabla$ the momentum operator, $\mathbf{A}$ is the potential (2) and the following representation of the Dirac $4 \times 4$ matrices is used

$\boldsymbol{\alpha}_{i}=\left(\begin{array}{cc}0 & \sigma_{i} \\ \sigma_{i} & 0\end{array}\right), \quad \boldsymbol{\beta}=\left(\begin{array}{cc}\mathrm{I} & 0 \\ 0 & -\mathrm{l}\end{array}\right)$

with I the $2 \times 2$ unit matrix and $\sigma_{i}$ the Pauli matrices [5]. Due to the symmetries of the magnetic field, we will use the natural cylindrical coordinates to express the stationary Dirac equation for the energy $E$,

$\mathbf{H} \Psi(\rho, \theta, z)=E \Psi(\rho, \theta, z)$.

We can separate this equation by looking for solutions of the following form:

$\Psi(\rho, \theta, z)=e^{i z \mu / \hbar} e^{i \lambda \theta / \hbar} e^{-i \theta \Sigma_{z} / 2 \hbar} \frac{1}{\sqrt{\rho}} \psi(\rho)$.

The translation symmetry in the $z$ direction has the symmetry generator $P_{z}=-i \hbar \frac{\partial}{\partial z}$, and the rotational symmetry around the $z$ axis is represented by the symmetry operator $J_{z}$ given by

$J_{z}=-i \hbar \frac{\partial}{\partial \theta}+\frac{1}{2} \hbar \Sigma_{z}, \quad \Sigma_{z}=\left(\begin{array}{cc}\sigma_{3} & 0 \\ 0 & \sigma_{3}\end{array}\right)$.
The above eigenfunction (11) is just a simultaneous eigenfunction of $P_{z}$ and $J_{z}$ with eigenvalues $\mu$ and $\lambda$, respectively,

$P_{z} \Psi(\rho, \theta, z)=\mu \Psi(\rho, \theta, z), \quad J_{z} \Psi(\rho, \theta, z)=\lambda \Psi(\rho, \theta, z)$.

Since we are dealing with an electron of spin $1 / 2$, the eigenvalues $\lambda$ of $J_{z}$ can take only half integer values: $\pm 1 / 2, \pm 3 / 2, \ldots$. Finally, in order to end with a Hermitian matrix equation, we have included the factor $1 / \sqrt{\rho}$ in (11).

Once this expression is replaced in the Dirac equation (10), the following reduced equation for $\psi(\rho)$ is obtained,

$$
\begin{aligned}
& \mathcal{H} \psi(\rho) \equiv \\
& {\left[-i \hbar c \alpha_{1} \frac{\partial}{\partial \rho}+\left(\frac{\lambda \hbar c}{\rho}-e k\right) \alpha_{2}+c \mu \alpha_{3}+m_{0} c^{2} \beta\right] \psi(\rho)} \\
& =E \psi(\rho)
\end{aligned}
$$

where the operator $\mathcal{H}$ in the variable $\rho$ is an effective Hamiltonian. This Hamiltonian, after the substitution of the $\boldsymbol{\alpha}_{i}, \boldsymbol{\beta}$ matrices, has a simple structure:

$\mathcal{H}=\left(\begin{array}{cc}m_{0} c^{2} \mathrm{I} & \mathrm{h} \\ \mathrm{h} & -m_{0} c^{2} \mathrm{I}\end{array}\right)$

where we have introduced another two-dimensional matrix Hamiltonian $\mathrm{h}$ defined by

$\mathrm{h}=-i \hbar c \sigma_{1} \frac{\partial}{\partial \rho}+\left(\frac{\lambda \hbar c}{\rho}-e k\right) \sigma_{2}+c \mu \sigma_{3}$.

Hereafter, in order to simplify the notation in the algebraic manipulations, we will take the following convention

$\hbar=c=1, \quad e k=\kappa$

(this is equivalent to take a new variable $\tilde{\rho}=\rho / \hbar c$ and parameter $\tilde{\mu}=c \mu$, but we will keep the notation without tildes). With this convention $\mathrm{h}$ has the form

$\mathrm{h}=\left(\begin{array}{cc}\mu & i a^{+} \\ -i a^{-} & -\mu\end{array}\right)$

where the scalar operators $a^{ \pm}$in the variable $\rho$ are given by

$a^{ \pm}=\mp \frac{\partial}{\partial \rho}-\frac{\lambda}{\rho}+\kappa$.

As these operators are formally adjoint of each other, $\left(a^{-}\right)^{\dagger}=a^{+}$, the operator $\mathrm{h}$ is Hermitian too.

\subsection{Eigenvalue problem of the effective Hamiltonian $\mathcal{H}$}

We have reduced the initial problem to finding the eigenfunctions of $\mathcal{H}$

$\mathcal{H} \psi=E \boldsymbol{\psi}, \quad \boldsymbol{\psi}=\left(\begin{array}{c}\varphi \\ \chi\end{array}\right)$

where $\varphi$ and $\chi$ are the two-components of $\psi$. With the help of (13), and having in mind that $c=1,(18)$ leads us to a coupled pair of equations,

$\left\{\begin{array}{l}m_{0} \varphi+\mathrm{h} \chi=E \varphi \\ \mathrm{h} \varphi-m_{0} \chi=E \chi\end{array} \quad \Longrightarrow \quad \chi=\frac{1}{m_{0}+E} \mathrm{~h} \varphi\right.$.

Next, by replacing this value of $\chi$, we get an equation for $\varphi$ :

$\mathrm{h}^{2} \varphi=\left(E^{2}-m_{0}^{2}\right) \varphi \equiv \varepsilon \varphi \Longrightarrow E_{ \pm}= \pm \sqrt{\varepsilon+m_{0}^{2}}$. 
The corresponding wave functions for the two energy signs differ in the coefficient of the second component,

$\chi_{ \pm}=\frac{1}{m_{0}+E_{ \pm}} \mathrm{h} \varphi$

and therefore the eigenfunctions have the form

$\boldsymbol{\psi}_{ \pm}=\left(\begin{array}{c}\varphi \\ \chi_{ \pm}\end{array}\right)$

From (16) we obtain an explicit expression of $h^{2}$,

$\mathrm{h}^{2}=\left(\begin{array}{cc}a^{+} a^{-}+\mu^{2} & 0 \\ 0 & a^{-} a^{+}+\mu^{2}\end{array}\right) \equiv\left(\begin{array}{cc}\mathrm{h}_{\uparrow}^{2} & 0 \\ 0 & \mathrm{~h}_{\downarrow}^{2}\end{array}\right)$

which is given in terms of the scalar Hamiltonians $h_{\uparrow \downarrow}^{2}$,

$\left\{\begin{array}{l}\mathrm{h}_{\uparrow}^{2}=a^{+} a^{-}+\mu^{2}=-\frac{\partial^{2}}{\partial \rho^{2}}+\frac{\lambda(\lambda-1)}{\rho^{2}}-\frac{2 \lambda \kappa}{\rho}+\kappa^{2}+\mu^{2} \\ \mathrm{~h}_{\downarrow}^{2}=a^{-} a^{+}+\mu^{2}=-\frac{\partial^{2}}{\partial \rho^{2}}+\frac{\lambda(\lambda+1)}{\rho^{2}}-\frac{2 \lambda \kappa}{\rho}+\kappa^{2}+\mu^{2} .\end{array}\right.$

In order to have bound states it is necessary that $\lambda \kappa>0$; hereafter we will assume that this is the case. We also assume that $\kappa>0$, so that the values of $\lambda$ must be greater or equal than $1 / 2$. The solutions of $h^{2}$ in (20) will have the form

$\varphi=\left(\begin{array}{c}\alpha f^{\uparrow} \\ \beta f^{\downarrow}\end{array}\right), \quad \alpha, \beta \in \mathbb{C}$

where each component $f^{\uparrow \downarrow}$ is a solution of the corresponding scalar Hamiltonian, with the same eigenvalue

$\mathrm{h}_{\uparrow}^{2} f^{\uparrow}=\varepsilon f^{\uparrow}, \quad \mathrm{h}_{\downarrow}^{2} f^{\downarrow}=\varepsilon f^{\downarrow}, \quad \varepsilon=E^{2}-m_{0}^{2}$.

The following relations of the spectrum and eigenfunctions of $h_{\uparrow \downarrow}^{2}$ will hold:

(1) The two scalar Hamiltonians $h_{\uparrow \downarrow}^{2}$ in (24) are supersymmetric partners corresponding to the Coulomb problem [6-9],

$$
a^{-} \mathrm{h}_{\uparrow}^{2}=\mathrm{h}_{\downarrow}^{2} a^{-}, \quad \mathrm{h}_{\uparrow}^{2} a^{+}=a^{+} \mathrm{h}_{\downarrow}^{2}
$$

(2) These scalar Hamiltonians $h_{\uparrow \downarrow}^{2}$ are almost isospectral. They have the same spectrum except for the ground state of $h_{\uparrow}^{2}$ which is not present in $h_{\downarrow}^{2}$ :

$\left\{\begin{array}{l}\mathrm{h}_{\uparrow}^{2} f_{n}^{\uparrow}=\varepsilon_{n} f_{n}^{\uparrow}, n \geq 0, \\ \mathrm{~h}_{\downarrow}^{2} f_{n}^{\downarrow}=\varepsilon_{n} f_{n}^{\downarrow}, n \geq 1,\end{array}\right.$

$\mu_{n}^{2} \equiv \varepsilon_{n}=\kappa^{2}+\mu^{2}-\frac{\kappa^{2} \lambda^{2}}{(\lambda+n)^{2}}$

The explicit form of the eigenfunctions is

$f_{n}^{\uparrow}(z)=N_{n}^{\uparrow} z^{(\lambda)} \exp (-z / 2) L_{n}^{(2 \lambda-1)}(z)$,

$f_{n}^{\downarrow}(z)=N_{n}^{\downarrow} z^{(\lambda+1)} \exp \left(-\frac{z}{2}\right) L_{n-1}^{(2 \lambda+1)}(z)$,

where $z=\frac{2 \kappa \lambda}{(\lambda+n)} \rho$. Here, $L_{n}^{(\alpha)}(z)$ designs the Laguerre polynomials and $N_{n}^{\uparrow \downarrow}$ are normalization constants. The isospectral eigenfunctions are related by means of the $a^{ \pm}$operators,

$\begin{cases}a^{-} f_{0}^{\uparrow}=0 & \\ a^{-} f_{n}^{\uparrow}=\sqrt{\varepsilon_{n}-\varepsilon_{0}} f_{n}^{\downarrow}, & n \geq 1 \\ a^{+} f_{n}^{\downarrow}=\sqrt{\varepsilon_{n}-\varepsilon_{0}} f_{n}^{\uparrow}, & n \geq 1\end{cases}$

\subsection{Solutions to the simultaneous eigenvalue problem of $\mathrm{h}^{2}$ and $\mathrm{h}$}

Once we have the eigenvalue solutions for the scalar Hamiltonians, the question of the eigenvalue problem of the diagonal Hamiltonian $\mathrm{h}^{2}$ of (25) and (26) can be straightforwardly answered,

$\mathrm{h}^{2} \varphi_{n}=\left(E^{2}-m_{0}^{2}\right) \varphi_{n}=\varepsilon_{n} \varphi_{n}$

with

$\varphi_{0}=\left(\begin{array}{c}f_{0}^{\uparrow} \\ 0\end{array}\right), \varphi_{n}=\left(\begin{array}{c}\alpha f_{n}^{\uparrow} \\ \beta f_{n}^{\downarrow}\end{array}\right), n \in \mathbb{N}, \alpha, \beta \in \mathbb{C}$

Each eigenvalue $\varepsilon_{n}, n \geq 1$ is doubly degenerated. We can distinguish two independent eigenfunctions by choosing them as the simultaneous eigenfunctions of $\mathrm{h}$ and $\mathrm{h}^{2}$ :

$\left\{\begin{array}{l}\mathrm{h} \varphi_{n}^{s}=s \sqrt{\varepsilon_{n}} \varphi_{n}^{s} \\ \mathrm{~h}^{2} \varphi_{n}^{s}=\varepsilon_{n} \varphi_{n}^{s}\end{array} \quad n \geq 1, \varepsilon_{n}=\mu_{n}^{2}, s= \pm\right.$.

The eigenvalue $\varepsilon_{0}$ has only one independent eigenfunction corresponding to positive eigenvalue of $h$,

$\left\{\begin{array}{l}\mathrm{h} \varphi_{0}=+\sqrt{\varepsilon_{0}} \varphi_{0} \\ \mathrm{~h}^{2} \varphi_{0}=\varepsilon_{0} \varphi_{0}\end{array} \quad n=0, \varepsilon_{0}=\mu^{2}\right.$.

Replacing (33) and (34) in (32) we obtain the coefficients $\alpha, \beta$ corresponding to each sign, for $n \geq 1$,

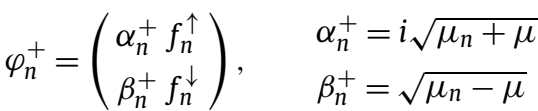

$\varphi_{n}^{-}=\left(\begin{array}{c}\alpha_{n}^{-} f_{n}^{\uparrow} \\ \beta_{n}^{-} f_{n}^{\downarrow}\end{array}\right), \quad \begin{aligned} & \alpha_{n}^{-}=i \sqrt{\mu_{n}-\mu} \\ & \beta_{n}^{-}=-\sqrt{\mu_{n}+\mu}\end{aligned}$

where $f_{n}^{\uparrow \downarrow}$ are related by the $a^{ \pm}$operators through (30) and $\mu_{n}$ is the positive root of $\mu_{n}^{2}$ defined in (28).

\subsection{The eigenfunctions and eigenvalues of $\mathcal{H}$}

Now, we have all the ingredients to write explicitly the eigenfunctions and eigenvalues of $\mathcal{H}$. Taking into account the values of $\varepsilon_{n}$ in (28), then according to (20) the eigenvalues of the energy are given by

$E_{ \pm n}= \pm \sqrt{\mu_{n}^{2}+m_{0}^{2}}= \pm \sqrt{\kappa^{2}+\mu^{2}-\frac{\kappa^{2} \lambda^{2}}{(\lambda+n)^{2}}+m_{0}^{2}}$.

As there are two signs for the energies, $E_{ \pm n}$, from each component, $\varphi_{n}^{s}$, there will be two eigenfunctions according to (21) and (22),

$\chi_{ \pm n}^{s}=\frac{s \mu_{n}}{m_{0}+E_{ \pm n}} \varphi_{n}^{s}, \quad s= \pm, n=1,2, \ldots$

$\chi_{ \pm 0}=\frac{\mu_{0}}{m_{0}+E_{ \pm 0}} \varphi_{0}$.

In summary, we have arrived to the following eigenfunctions and eigenvalues,

$\boldsymbol{\psi}_{ \pm n}^{s}=\left(\begin{array}{c}\varphi_{n}^{s} \\ \chi_{ \pm n}^{s}\end{array}\right), E_{ \pm n}= \pm \sqrt{\mu_{n}^{2}+m_{0}^{2}}, n \geq 1, s= \pm$.

The two signs \pm in the subindex corresponds to signs of the corresponding energy; the two values $s$ of the superindex indicates the double degeneracy of each (positive or negative) eigenvalue. For the lowest value $n=0$, the eigenfunctions of the ground-levels are non-degenerated, so the superindex $s$ is missing,

$\boldsymbol{\psi}_{ \pm 0}=\left(\begin{array}{c}\varphi_{0} \\ \chi_{ \pm 0}\end{array}\right), E_{ \pm 0}= \pm \sqrt{\mu^{2}+m_{0}^{2}}, \quad n=0$. 


\subsection{Remarks on the spectrum}

(a) Spectrum of the reduced Hamiltonian $\mathcal{H}$. For fixed values of $\lambda, \mu$, the discrete spectrum of the one-dimensional effective Hamiltonian $\mathcal{H}$ has doubly degenerate excited levels $n=1,2, \ldots$. A pair of independent eigenfunctions are given by $\boldsymbol{\psi}_{ \pm n}^{s}$, labeled by the superindex $s= \pm$. This seems reasonable, since the total angular momentum around the $z$-axis $J_{z}=L_{z}+S_{z}$, is the sum of the orbital $L_{z}$ (with eigenvalues $\ell$ ) and spin $S_{z}$ momentum (with eigenvalues $\sigma$ ). Hence, there are two types of states which can contribute to produce $\lambda$-eigenfunctions: states with $\ell=\lambda+1 / 2$ and $\sigma=-1 / 2$ or states with $\ell=\lambda-1 / 2$ and $\sigma=1 / 2$. Thus, as the $\lambda$-eigenfunctions belong to this subspace of dimension two, each energy level can be doubly degenerated.

However, in the case of the ground states, the eigenfunctions with total momentum $\lambda$ are made up of $\varphi_{0}$ and $\chi_{ \pm 0}$ which are eigenfunctions of $S_{z}$ with eigenvalue $1 / 2$. In this special case the ground eigenfunctions are at the same time eigenfunctions of the orbital $L_{z}$ and spin $S_{z}$ momentum with eigenvalues $\ell=\lambda-1 / 2$ and $1 / 2$, respectively. In this situation the degeneration has been lost and the ground states with energies $E_{ \pm 0}$ are not degenerate. These ground states must have spin aligned with the magnetic field.

(b) Spectrum of the total Hamiltonian with $\mu$ fixed. Now, let us pay attention to the energy eigenvalues for different values of the total angular momentum $\lambda$ given in (36):

$E_{ \pm n}= \pm \sqrt{m_{0}^{2}+\mu^{2}+\kappa^{2}-\frac{\kappa^{2}}{(1+n / \lambda)^{2}}}$,

$n=0,1 \ldots, \lambda=1 / 2,3 / 2, \ldots$

If we consider this problem restricted to the plane, these energies correspond to the discrete spectrum of bound states. The square of such energies is bounded by two fixed values:

$m_{0}^{2}+\mu^{2} \leq\left(E_{ \pm n}\right)^{2}<m_{0}^{2}+\mu^{2}+\kappa^{2}$.

The most important aspect here is that, the (square of the) energy spectrum consist in a dense subset of points in the closed interval $\left[m_{0}^{2}+\mu^{2}, m_{0}^{2}+\mu^{2}+\kappa^{2}\right]$. In particular, there is no isolated point in the spectrum of bound states! This is quite different from the discrete spectrum of superintegrable systems which have a discrete spectrum made of isolated points, as it happens with the planar Coulomb potential (which has a similar dependence on $\rho$ ) or the Landau system in the plane (for a uniform magnetic field).

(c) Degeneracy of energy levels. The second important feature worth to remark is that each energy level (fixed for instance by a pair of values $n_{0}$ and $\lambda_{0}$ ) has an infinite degeneracy given by all the values of $\lambda$ and $n$ such that $\lambda / n=\lambda_{0} / n_{0}$. Usually, the degeneracy of energy levels is associated with superintegrable systems, such as the above mentioned Coulomb or Landau systems. The argument is that the additional symmetries of such systems imply this nontrivial degeneracy. This is correct, but it does not mean that integrable systems have no degeneracy at all, as the present example shows in a drastic way.

\section{Shape-invariance}

In the study of the problem of an electron immersed in a nonuniform magnetic field, we have arrived to an effective matrix Hamiltonian in one variable $\mathcal{H}$, which is exactly solvable. Now, we want to know whether, as it happens with some exactly solvable one-dimensional scalar Hamiltonians, the matrix Hamiltonian $\mathcal{H}$ takes part in a hierarchy of Hamiltonians $\mathcal{H}_{n}, n \in \mathbb{Z}$ which are shape-invariant. In other words, we ask if there is a sequence $\mathcal{H}_{n}$ together with a set of matrix differential operators $A_{n}$ such that
$\mathcal{H}_{n} A_{n}^{+}=A_{n}^{+} \mathcal{H}_{n+1} \Longleftrightarrow A_{n}^{-} \mathcal{H}_{n}=\mathcal{H}_{n+1} A_{n}^{-}$

where $A_{n}^{+}$is the adjoint differential operator of $A_{n}^{-}$and $\mathcal{H}$ is identified with $\mathcal{H}_{0}$. We will show that the answer is positive. As in the scalar shape-invariant case, the spectrum and eigenfunctions of $\mathcal{H}$ will be obtained from the energy and ground states of the sequence $\mathcal{H}_{n}$. Then, we can say that in this sense our system is algebraically solvable. At the same time, we want to investigate some of the main differences between scalar and matrix shapeinvariance by means of the present case.

\subsection{Hamiltonian hierarchy}

In the following we will restrict ourselves to show the answer to this problem, obtained after some lengthy but straightforward computations. First of all, we write down the structure of the initial Hamiltonian. From (13), (16) and (17) we have the expressions,

$\mathcal{H}=\left(\begin{array}{cc}m_{0} \mathrm{I} & \mathrm{h} \\ \mathrm{h} & -m_{0} \mathrm{I}\end{array}\right), \quad \mathrm{h}=\left(\begin{array}{cc}\mu & i a^{+} \\ -i a^{-} & -\mu\end{array}\right)$,

$a^{ \pm}=\mp \frac{\partial}{\partial \rho}-\frac{\lambda}{\rho}+\kappa$.

The operators $a^{ \pm}$take part in the scalar hierarchy $a_{n}^{ \pm}$of the Coulomb problem [6-9] given by

$a_{n}^{ \pm}=\mp \frac{\partial}{\partial \rho}-\frac{(\lambda+n)}{\rho}+\frac{\lambda}{(\lambda+n)} \kappa$,

satisfying

$a_{n}^{-} a_{n}^{+}+\mu_{n}^{2}=a_{n+1}^{+} a_{n+1}^{-}+\mu_{n+1}^{2}$.

Therefore, we can look at (42) as the first Hamiltonian, for $n=0$, of a "natural" hierarchy, $\mathcal{H}_{n}$, obtained by means of the scalar operators $a_{n}^{ \pm}$:

$\mathcal{H}_{n}=\left(\begin{array}{cc}m_{0} \mathrm{I} & \mathrm{h}_{n} \\ \mathrm{~h}_{n} & -m_{0} \mathrm{l}\end{array}\right), \mathrm{h}_{n}=\left(\begin{array}{cc}\mu_{n} & i a_{n}^{+} \\ -i a_{n}^{-} & -\mu_{n}\end{array}\right)$

which include the parameters $\lambda_{n}, \kappa_{n}$ and $\mu_{n}$ defined by

$\lambda_{n}=\lambda+n, \kappa_{n}=\frac{\lambda}{(\lambda+n)} \kappa, \kappa_{n}^{2}+\mu_{n}^{2}=\kappa^{2}+\mu^{2}$.

\subsection{Basic intertwining operators}

Before writing the intertwining operators for the $4 \times 4$ Hamiltonians $\mathcal{H}_{n}$, we will consider this problem for the component $2 \times 2$ Hamiltonians $h_{n}$. We have found two different intertwining operators: $\mathrm{R}_{n}^{-}$and $\mathrm{T}_{n}^{-}$, which realize the intertwining with different signs,

$$
\begin{gathered}
\mathrm{R}_{n}^{-} \mathrm{h}_{n}=\mathrm{h}_{n+1} \mathrm{R}_{n}^{-} \quad \Longleftrightarrow \quad \mathrm{h}_{n} \mathrm{R}_{n}^{+}=\mathrm{R}_{n}^{+} \mathrm{h}_{n+1} \\
\mathrm{~T}_{n}^{-} \mathrm{h}_{n}=-\mathrm{h}_{n+1} \mathrm{~T}_{n}^{-} \quad \Longleftrightarrow \quad \mathrm{h}_{n} \mathrm{~T}_{n}^{+}=-\mathrm{T}_{n}^{+} \mathrm{h}_{n+1},
\end{gathered}
$$

where $\mathrm{R}_{n}^{+}=\left(\mathrm{R}_{n}^{-}\right)^{\dagger}$, and $\mathrm{T}_{n}^{+}=\left(\mathrm{T}_{n}^{-}\right)^{\dagger}$. They have the following expressions:

$\mathrm{R}_{n}^{-}=\left(\begin{array}{cc}a_{n}^{-} & -i\left(\mu_{n}-\mu_{n+1}\right) \\ 0 & a_{n+1}^{-}\end{array}\right)$
$\mathrm{T}_{n}^{-}=\left(\begin{array}{cc}a_{n}^{-} & -i\left(\mu_{n}+\mu_{n+1}\right) \\ 0 & -a_{n+1}^{-}\end{array}\right)$.

The proof consists in a straightforward checking. The intertwining with minus sign (or anti-intertwining) in (45) has not been considered before, but it can be present in Dirac-type Hamiltonians whose spectrum contains positive and negative values. While 
$R_{n}^{-}\left(R_{n}^{+}\right)$connect eigenstates from $h_{n}\left(h_{n+1}\right)$ to $h_{n+1}\left(h_{n}\right)$ with the same eigenvalue, the operators $\mathrm{T}_{n}^{ \pm}$relate eigenstates with opposite energy. From their explicit form (46), it is easy to compute the kernel eigenspaces $\mathrm{K}_{n}$ and $\tilde{\mathrm{K}}_{n}$ of $\mathrm{h}_{n}$ annihilated by $\mathrm{R}_{n}^{-}$or $\mathrm{T}_{n}^{-}$ respectively,

$\mathrm{R}_{n}^{-} \varphi=0 \Longrightarrow \varphi \in \mathrm{K}_{n}:=\left\langle{ }_{n} \varphi_{0},{ }_{n} \varphi_{1}^{-}\right\rangle$,

eigenvalues of $\mathrm{h}_{n}:\left\{\mu_{n},-\mu_{n+1}\right\}$

$\mathrm{T}_{n}^{-} \varphi=0 \Longrightarrow \varphi \in \tilde{\mathrm{K}}_{n}:=\left\langle n \varphi_{0},{ }_{n} \varphi_{1}^{+}\right\rangle$,

eigenvalues of $h_{n}:\left\{\mu_{n}, \mu_{n+1}\right\}$

where ${ }_{n} \varphi_{k}^{ \pm}$denote the eigenfunctions of $h_{n}$ corresponding to its $\pm k$-th excited states. In other words, $\mathrm{R}_{n}^{-}$annihilates the positive and the negative ground states of $h_{n}$. However, $T_{n}^{-}$will annihilate the positive ground state and the first positive excited state of $h_{n}$. We can get the whole discrete set of eigenfunctions of $h_{0}$ from any of these two types of eigenfunction sets of $h_{n}, n=0,1,2, \ldots$, and the iterative use of $\mathrm{R}_{n}^{+}$or $\mathrm{T}_{n}^{+}$. For example, we can get the following excited states of $h_{n-1}$ from those of $h_{n}$,

$\mathrm{R}_{n-1}^{+}:{ }_{n} \varphi_{0} \rightarrow{ }_{n-1} \varphi_{1}^{+}, \quad \mathrm{R}_{n-1}^{+}:{ }_{n} \varphi_{1}^{-} \rightarrow{ }_{n-1} \varphi_{2}^{-}$

$\mathrm{T}_{n-1}^{+}:{ }_{n} \varphi_{0} \rightarrow{ }_{n-1} \varphi_{1}^{-}, \quad \mathrm{T}_{n-1}^{+}:{ }_{n} \varphi_{1}^{+} \rightarrow_{n-1} \varphi_{2}^{-}$.

\subsection{General intertwining operators}

From the basic $2 \times 2$ intertwining operators $\mathrm{R}_{n}^{-}$, $\mathrm{T}_{n}^{-}$, we can construct the 'global intertwining' $4 \times 4$ operators (41) of the Hamiltonian hierarchy $\mathcal{H}_{n}$. We have obtained four linearly independent operators:

$\mathcal{R}_{n}^{-}=\left(\begin{array}{cc}\mathrm{R}_{n}^{-} & 0 \\ 0 & \mathrm{R}_{n}^{-}\end{array}\right), \mathcal{T}_{n}^{-}=\left(\begin{array}{cc}-\mathrm{T}_{n}^{-} & 0 \\ 0 & \mathrm{~T}_{n}^{-}\end{array}\right)$,

$\widetilde{\mathcal{R}}_{n}^{-}=\left(\begin{array}{cc}-\mathrm{M} & \mathrm{R}_{n}^{-} \\ \mathrm{R}_{n}^{-} & \mathrm{M}\end{array}\right), \widetilde{\mathcal{T}}_{n}^{-}=\left(\begin{array}{cc}\mathrm{M} & -\mathrm{T}_{n}^{-} \\ \mathrm{T}_{n}^{-} & \mathrm{M}\end{array}\right)$.

The constant matrix $\mathrm{M}=m_{0}\left(-i \sigma_{1}+\sigma_{2}\right)=2 m_{0} \sigma^{-}$satisfies the following commutation rules

$\boldsymbol{\sigma}^{-} \mathrm{h}_{n}+\mathrm{h}_{n+1} \boldsymbol{\sigma}^{-}=-\mathrm{R}_{n}, \quad \boldsymbol{\sigma}^{-} \mathrm{h}_{n}-\mathrm{h}_{n+1} \boldsymbol{\sigma}^{-}=-\mathrm{T}_{n}$.

It is straightforward to check that, indeed the operators (49), with the help of (50), satisfy the intertwining relation (41). Since any of the operators in (49) intertwines the hierarchy, the same happens for any linear combination of them. Therefore, we have obtained a four dimensional vector space of intertwining operators for the same hierarchy.

\subsection{Symmetries}

A symmetry of $\mathcal{H}_{n}$ is a differential operator $\mathcal{S}_{n}$ such that

$\mathcal{H}_{n} \mathcal{S}_{n}=\mathcal{S}_{n} \mathcal{H}_{n}$

In principle, we are interested in the basic symmetry operators: up to first order matrix differential operators. As the equation (51) is similar to the intertwining relation (41) we can obtain all the solutions in the same way. We have found (besides the trivial unit matrix and the Hamiltonian itself) four such symmetry operators which are linearly independent:

$\mathcal{S}_{n}^{1}=\left(\begin{array}{cc}\mathrm{h}_{n} & 0 \\ 0 & \mathrm{~h}_{n}\end{array}\right), \quad \mathcal{S}_{n}^{2}=\left(\begin{array}{cc}m_{0} \sigma_{3} & \mu_{n} \mathrm{I} \\ \mu_{n} \mathrm{I} & -m_{0} \sigma_{3}\end{array}\right)$

$\mathcal{S}_{n}^{3}=\left(\begin{array}{cc}-m_{0} \sigma_{3} & -\sigma_{3} \mathrm{~h}_{n}+\mu_{n} \mathrm{l} \\ \sigma_{3} \mathrm{~h}_{n}-\mu_{n} \mathrm{l} & -m_{0} \sigma_{3}\end{array}\right)$,

$\mathcal{S}_{n}^{4}=\left(\begin{array}{cc}-\sigma_{3} \mathrm{~h}_{n}+\mu_{n} \mathrm{l} & 0 \\ 0 & \sigma_{3} \mathrm{~h}_{n}-\mu_{n} \mathrm{l}\end{array}\right)$
One can easily check that the above operators are symmetries having in mind that the matrix $\sigma_{3}$ satisfies the following property

$\sigma_{3} h_{n}+h_{n} \sigma_{3}=2 \mu_{n}$.

Although linearly independent, the last two symmetries can be obtained as an algebraic function of the first three:

$\mathcal{S}_{n}^{3}=-\frac{1}{m_{0}}\left(\mathcal{S}_{n}^{2} \mathcal{H}_{n}-\mu_{n} \mathcal{S}_{n}^{1}\right), \quad \mathcal{S}_{n}^{4}=-\frac{1}{m_{0}}\left(\mathcal{S}_{n}^{1} \mathcal{S}_{n}^{2}-\mu_{n} \mathcal{H}_{n}\right)$.

The symmetries $\mathcal{S}^{2}$ and $\mathcal{S}^{3}$ do not commute and the Hamiltonian is obtained from their anticommutator:

$\left\{\mathcal{S}_{n}^{2}, \mathcal{S}_{n}^{3}\right\}=2 \mu_{n} \mathcal{H}_{n}$

These symmetries are quite important in our problem. For instance, the eigenfunctions given in (38) and (39) are characterized by being also eigenfunctions of $\mathcal{S}^{2}$. Another equivalent set of solutions could be obtained by the simultaneous eigenfunctions of $\mathcal{H}_{n}$ and $\mathcal{S}^{1}$.

There is a simple and useful relation between symmetry and intertwining operators that we will consider in the following. Let $\mathcal{H}_{0}$ and $\mathcal{H}_{1}$ be two Hamiltonians, and $\mathcal{A}^{+}, \mathcal{B}^{-}$be intertwining operators such that $\mathcal{B}^{-} \mathcal{H}_{0}=\mathcal{H}_{1} \mathcal{B}^{-}$and $\mathcal{H}_{0} \mathcal{A}^{+}=\mathcal{A}^{+} \mathcal{H}_{1}$. Let also $\mathcal{S}_{0}$ and $\mathcal{S}_{1}$ be symmetries of $\mathcal{H}_{0}$ and $\mathcal{H}_{1}$, respectively. Then:

(i) $\mathcal{A}^{+} \mathcal{B}^{-}$is a symmetry of $\mathcal{H}_{0}$, and $\mathcal{B}^{-} \mathcal{A}^{+}$is a symmetry of $\mathcal{H}_{1}$.

(ii) The operators $\mathcal{S}_{0} \mathcal{A}^{+}$and $\mathcal{A}^{+} \mathcal{S}_{1}$ are intertwining operators in the same sense as $\mathcal{A}^{+}$. The products of operators $\mathcal{B}^{-} \mathcal{S}_{0}$ and $\mathcal{S}_{1} \mathcal{B}^{-}$are intertwining operators playing the same role as $\mathcal{B}^{-}$.

All the intertwining operators given in (49) are related in this way with the symmetries of the Hamiltonians $\mathcal{H}_{n}$.

\subsection{Spectrum and eigenfunctions from intertwining operators}

Let us consider one of the intertwining operators, for instance $\mathcal{R}_{n}^{-}$, satisfying the relation $\mathcal{R}_{n}^{-} \mathcal{H}_{n}=\mathcal{H}_{n+1} \mathcal{R}_{n}^{-}$. Let us call $\mathcal{K}_{n}$ to the kernel of $\mathcal{R}_{n}^{-}$, in other words,

$\mathcal{K}_{n}=\left\{\boldsymbol{\psi} ; \mathcal{R}_{n}^{-} \boldsymbol{\psi}=\mathbf{0}\right\}$

Then, it is immediate to show from the above intertwining relation that $\mathcal{K}_{n}$ is an invariant subspace of $\mathcal{H}_{n}$. $\mathcal{K}_{n}$ is a four dimensional space, a basis made up of eigenvectors of $\mathcal{H}_{n}$ in this space is easily computed from (53):

$\mathcal{K}_{n}=\left\langle{ }_{n} \psi_{+0},{ }_{n} \boldsymbol{\psi}_{-0},{ }_{n} \boldsymbol{\psi}_{+1}^{-},{ }_{n} \boldsymbol{\psi}_{-1}^{-}\right\rangle$

where we have extended the notation given in (38)-(39) to the eigenfunctions of $\mathcal{H}_{n}$,

${ }_{n} \boldsymbol{\psi}_{ \pm 0}=\left(\begin{array}{c}{ }_{n} \varphi_{0} \\ { }_{n} \chi_{ \pm 0}\end{array}\right), \quad{ }_{n} \boldsymbol{\psi}_{ \pm 1}^{-}=\left(\begin{array}{c}{ }_{n} \varphi_{1}^{-} \\ { }_{n} \chi_{ \pm 1}^{-}\end{array}\right)$.

The eigenvalues corresponding to these states are, respectively,

${ }_{n} E_{ \pm 0}= \pm \sqrt{\mu_{n}^{2}+m_{0}^{2}}, \quad{ }_{n} E_{ \pm 1}= \pm \sqrt{\mu_{n+1}^{2}+m_{0}^{2}}$.

These are four of the lower energy states of Hamiltonian $\mathcal{H}_{n}$, two of them in the positive spectrum and the other two in the negative one.

Next, we will show that from the four independent states of the kernel (54) we can build four excited states of $\mathcal{H}_{0}$ by means of the operators $\mathcal{R}_{n}^{+}$in the usual way:

${ }_{0} \boldsymbol{\psi}_{ \pm n}^{+}=\mathcal{R}_{0}^{+} \mathcal{R}_{1}^{+} \ldots \mathcal{R}_{(n-1)}^{+} n \boldsymbol{\psi}_{ \pm 0}$
${ }_{0} \boldsymbol{\psi}_{ \pm(n+1)}^{-}=\mathcal{R}_{0}^{+} \mathcal{R}_{1}^{+} \ldots \mathcal{R}_{(n-1)}^{+}{ }^{+} \boldsymbol{\psi}_{ \pm 1}^{-}$. 
As each energy level of $\mathcal{H}_{0}$ is two-fold degenerate (except the two ground levels) the other independent states of the above four excited levels can be obtained in a similar way from other Hamiltonians in the same hierarchy. Explicitly,

$$
\begin{aligned}
& { }_{0} \boldsymbol{\psi}_{ \pm n}^{-}=\mathcal{R}_{0}^{+} \mathcal{R}_{1}^{+} \ldots \mathcal{R}_{(n-2)}^{+}(n-1) \boldsymbol{\psi}_{ \pm 1}^{-} \\
& { }_{0} \boldsymbol{\psi}_{ \pm(n+1)}^{+}=\mathcal{R}_{0}^{+} \mathcal{R}_{1}^{+} \ldots \mathcal{R}_{n}^{+}{ }_{(n+1)} \boldsymbol{\psi}_{ \pm 0} .
\end{aligned}
$$

We can check that indeed, these new states (58) and (59) are orthogonal to the previous ones (56) and (57), respectively. Take for instance the following inner product

$$
\left\langle\mathcal{R}_{0}^{+} \ldots \mathcal{R}_{(n-1)}^{+} n \boldsymbol{\psi}_{ \pm 0}, \mathcal{R}_{0}^{+} \ldots \mathcal{R}_{(n-2)}^{+}(n-1) \boldsymbol{\psi}_{ \pm 1}^{1}\right\rangle \text {. }
$$

Then, by making use of the adjoint of the operators $\mathcal{R}_{n}^{+}$, this can be shown to be proportional to

$\left\langle\mathcal{R}_{(n-1)}^{+} n \boldsymbol{\psi}_{ \pm 0},{ }_{(n-1)} \boldsymbol{\psi}_{ \pm 1}^{1}\right\rangle=\left\langle{ }_{n} \boldsymbol{\psi}_{ \pm 0}, \mathcal{R}_{(n-1)}^{-}(n-1) \boldsymbol{\psi}_{ \pm 1}^{1}\right\rangle=0$

The vanishing of the last term comes from the fact that ${ }_{(n-1)} \boldsymbol{\psi}_{ \pm 1}^{1}$ is in the kernel of $R_{(n-1)}^{-}$. In this way all the eigenfunctions of the initial Hamiltonian $\mathcal{H}_{0}$ can be obtained from the 'lowest energy' levels of the Hamiltonians in the hierarchy.

\subsection{Remarks on the matrix intertwining operators}

As we have seen, the intertwining operators, such as $\mathcal{R}_{n}^{-}$, are characterized by the annihilation of four of the 'lowest levels' of the system. Two of such levels are the ground (positive and negative) states, ${ }_{n} \boldsymbol{\psi}_{+0}$ and ${ }_{n} \boldsymbol{\psi}_{-0}$, which are non-degenerated. The other two must be selected from the first (positive and negative) excited levels. However, there is some freedom since each of these levels is doubly degenerated. The intertwining operators found in (49) correspond to choosing one positive and one negative eigenfunctions, this gives a set of four linearly independent intertwining operators. In general terms, the intertwining operators of matrix systems behave in a similar way to the usual scalar systems. They connect shape-invariant Hamiltonians, and generate the spectrum of one of the Hamiltonians from the ground states of the hierarchy. A difference, worth to stress, with scalar shape-invariance is that in the case of Dirac equations the intertwining relation can include a negative sign, as we have seen above for the basic $2 \times 2$ case, so that the intertwining operator will connect the positive and negative sectors.

In many superintegrable systems the algebraic solvability, in the sense of shape-invariance, can be applied but the intertwining operators have a different character. In our case, where the system is only integrable, the intertwining operators connect Hamiltonians with different parameters given in (44) by $\lambda_{n}, \kappa_{n}$ and $\mu_{n}$. To have a new system with values $\lambda_{n}, \mu_{n}$ instead of $\lambda_{0}, \mu_{0}$ is not quite important: these values belong to different constants of motion of the same system. However, the change of $\kappa_{0}$ by $\kappa_{n}$ means that these intertwining operators will connect different systems, because $\kappa$ measures the intensity of the external magnetic field. On the contrary, the Hamiltonian hierarchies of reduced superintegrable Hamiltonians are characterized by different values of constants of motion and the origin of intertwining operators are related to the additional symmetries. This is the case of the scalar Coulomb problem where the Runge-Lenz symmetries produce the intertwining of the reduced radial Hamiltonians corresponding to different angular momenta.

\section{Conclusions}

In this work we have investigated an integrable, but not superintegrable, system. Although this system is initially defined in the three dimensional space, the dynamics and relevant physical properties can be described by its reduction to a planar system. The non-superintegrability is characterized in the classical case by non-periodic trajectories in the plane which are dense in a domain. One of the main objectives of our paper was to address the question of the properties specific to a quantum non-superintegrable system. In the absence of trajectories, one must look for some special features of the spectrum and eigenfunctions. In our case we have seen that there are two such properties: (i) a dense character of the discrete spectrum (when the system is restricted to the plane) and (ii) an algebraic solvability involving Hamiltonians $\mathcal{H}_{n}$ of different problems. However, we saw that our quantum integrable system has a high degeneracy of energy levels, so that this property is not exclusive of superintegrability.

We have shown how the effective radial matrix Hamiltonian belongs to a hierarchy of shape-invariant matrix Hamiltonians. In this respect, we should mention that there are in the literature some examples of solvable Dirac systems in the context of the Dirac oscillator [11] or in the Jaynes-Cummings model [12]. The problem of Darboux transformations (or intertwining transformations) of Dirac Hamiltonians has been previously considered in [10] but restricted to two-component wave functions, or in [13] (from a very different point of view than in our approach). The shapeinvariance for matrix Hamiltonians of Schrödinger-Pauli type has been studied in $[14,15]$ and their Darboux properties in $[16,17]$ and references therein. However, in our case we have characterized some interesting features not found before, which can be summarized in the following points: i) the Hamiltonians in the hierarchy have degenerate energy levels; ii) this implies that the intertwining operators are non-unique, the freedom depending on the dimension of the degeneracy; iii) the non-trivial symmetry operators of each Hamiltonian have been obtained; iv) anti-intertwining operators are natural in the context of Dirac Hamiltonians.

\section{Acknowledgements}

We acknowledge financial support from Spanish MINECO, project MTM2014-57129-C2-1-P. ACA acknowledges CONACYT fellowship 207577.

\section{References}

[1] W. Miller Jr., S. Post, P. Winternitz, Classical and quantum superintegrability with applications, J. Phys. A 46 (2013) 423001.

[2] D. Bonatsos, C. Daskaloyannis, K. Kokkotas, Deformed oscillator algebras for two-dimensional quantum superintegrable systems, Phys. Rev. A 50 (1994) 3700.

[3] M. Kibler, P. Winternitz, Periodicity and quasi periodicity for superintegrable Hamiltonian systems, Phys. Lett. A 147 (1990) 338.

[4] A. Contreras-Astorga, D.J. Fernández, J. Negro, Solutions of the Dirac equation in a magnetic field and intertwining operators, SIGMA 8 (2012) 082, arXiv:1210.7416.

[5] J.D. Bjorken, S.D. Drell, Relativistic Quantum Mechanics, McGraw-Hill, 1964. [6] L. Infeld, T.E. Hull, The factorization method, Rev. Mod. Phys. 23 (1951) 21.

[7] C.V. Sukumar, Supersymmetry and the Dirac equation for a central Coulomb field, J. Phys. A 18 (1985) L697.

[8] O. Rosas-Ortiz, R. Muñoz, Non-Hermitian SUSY hydrogen-like Hamiltonians with real spectra, J. Phys. A 36 (2003) 8497.

[9] F. Cooper, A. Khare, U. Sukhatme, Supersymmetry and quantum mechanics, Phys. Rep. 251 (1995) 267, arXiv:hep-th/9405029.

[10] L.M. Nieto, A.A. Pecheritsin, B.F. Samsonov, Intertwining technique for the onedimensional stationary Dirac equation, Ann. Phys. 305 (2003) 151, arXiv:quantph/0307152.

[11] O. Castaños, A. Frank, R. López, L.F. Urrutia, Soluble extensions of the Dirac oscillator with exact and broken supersymmetry, Phys. Rev. D 43 (1991) 544

[12] V. Hussin, S. Kuru, J. Negro, Generalized Jaynes-Cummings Hamiltonians by shape-invariant hierarchies and their SUSY partners, J. Phys. A 39 (2006) 11301.

[13] E. Pozdeeva, A. Schulze-Halberg, Darboux transformations for a generalized Dirac equation in two dimensions, J. Math. Phys. 51 (2010) 113501, arXiv:0904. 0992. 
[14] M.V. Ioffe, S. Kuru, J. Negro, L.M. Nieto, SUSY approach to Pauli Hamiltonians with an axial symmetry, J. Phys. A, Math. Gen. 39 (2006) 6987, arXiv:hep-th/ 0603005.

[15] A.G. Nikitin, Y. Karadzhov, Enhanced classification of matrix superpotentials, J. Phys. A 44 (2011) 445202, arXiv:1107.2525.
[16] A.A. Andrianov, F. Cannata, M.V. Ioffe, D.N. Nishnianidze, Matrix Hamiltonians: SUSY approach to hidden symmetries, J. Phys. A 30 (1997) 5037.

[17] A.V. Sokolov, Spectral design for matrix Hamiltonians: different methods of constructing of a matrix intertwining operator, J. Phys. A 48 (2015) 085202. 